\title{
Estudio de la velocidad desarrollada por vehículos pesados en carreteras convencionales
}

\author{
Ana María Pérez Zuriaga \\ Grupo de Investigación en Ingeniería de Carreteras (GIIC), Universtitat Politècnica de \\ València \\ Francisco Javier Camacho Torregrosa, David Llopis Castelló, Sheila Ferrer López, \\ Alfredo García \\ GIIC, Universitat Politècnica de València
}

\section{RESUMEN}

La velocidad de operación, definida como el percentil 85 de la distribución de velocidad de vehículos ligeros circulando en carreteras convencionales en condiciones de flujo libre, es una de las principales variables en la evaluación de la consistencia del diseño geométrico. De esta forma, los únicos vehículos considerados en el análisis son los vehículos ligeros, obviando la influencia de los vehículos pesados. Para la estimación de la velocidad de operación de estos vehículos el número de modelos existentes es mínimo.

Por ello, el objetivo del trabajo que se presenta es el estudio experimental de la velocidad desarrollada por vehículos pesados en carreteras convencionales. El estudio no se centra únicamente en la velocidad de operación, sino en el estudio de la distribución completa de la velocidad, ya que los percentiles bajos están relacionados con el riesgo de alcance de vehículos ligeros. Se han analizado las diferentes variables que pueden influir con especial énfasis en las variables geométricas de la carretera.

El estudio se ha basado en los datos obtenidos gracias a la colaboración de dos empresas, que han permitido la colocación de dispositivos GPS de $1 \mathrm{~Hz}$ en vehículos de su flota. Los GPS registraron de forma continua la velocidad desarrollada por los vehículos durante la realización de su ruta habitual. La influencia sobre el comportamiento de los conductores fue mínima al tratarse de un dispositivo de pequeño tamaño ubicado en el exterior del vehículo.

Tras la restitución de la geometría de las carreteras objeto de estudio y el tratamiento de los datos de velocidad registrados, se analizó la influencia de las variables geométricas del trazado, especialmente en cuanto a alzado se refiere, en la velocidad. Se ha concluido que la pendiente no tiene una influencia significativa en la elección de la velocidad por parte de los conductores de vehículos pesados en curvas de carreteras convencionales, donde el principal control de la velocidad son los radios de las propias curvas.

\section{INTRODUCCIÓN}

El diseño geométrico de carreteras se ha basado tradicionalmente en la velocidad de proyecto 
y en la velocidad específica, asumiendo que los vehículos circulaban a esta velocidad o a una velocidad inferior. Sin embargo, se ha comprobado que esto no ocurre y que la velocidad de operación es muy distinta. Por ello, la tendencia en el diseño de carreteras es centrar el diseño de su trazado en la velocidad de operación estimada de los vehículos, definida por la AASHTO (2011) como aquella velocidad a la que circulan los vehículos ligeros en condiciones de flujo libre.

Para la estimación de la velocidad de operación desarrollada por vehículos ligeros $\left(\mathrm{V}_{85 \mathrm{~L}}\right)$ en carreteras convencionales existe una gran cantidad de modelos considerando unas $\mathrm{u}$ otras variables del trazado en planta o en alzado. Sin embargo, el número de modelos para la estimación de la velocidad de operación de vehículos pesados $\left(\mathrm{V}_{85 \mathrm{P}}\right)$ es muy reducido.

De hecho, en muchas ocasiones durante el diseño de carreteras se consideran los vehículos pesados únicamente para el diseño del firme, sin tenerlos en cuenta en aspectos tan importantes del diseño geométrico como es la consistencia del mismo. No considerar su velocidad de operación puede llevar a diseños geométricos inconsistentes. Harwood et al. (2003) subrayó que el diferencial de velocidad entre los vehículos ligeros y pesados puede producir que la operación de los vehículos en tramos de fuerte pendiente sea inconsistente. En 1977, Leisch y Leisch recomendaron para la obtención de un diseño geométrico consistente una diferencia máxima de $15 \mathrm{~km} / \mathrm{h}$ entre la velocidad de operación de los vehículos ligeros y los pesados desarrollada en un determinado elemento.

El desarrollo de un estudio en profundidad de la velocidad de operación de los vehículos pesados en tramos de fuerte pendiente es esencial para conseguir diseños consistentes para ambos tipos de vehículos. Por ello, algunas investigaciones han abordado el tema de la velocidad de operación de los vehículos pesados y sus diferencias con la de los vehículos ligeros, así como su influencia en la evaluación de la consistencia del diseño geométrico de carreteras.

Misaghi y Hassan (2005), tras el estudio de 40 curvas con diferentes características geométricas seleccionadas entre las existentes en 4 tramos diferentes de carreteras convencionales, consideraron que no había diferencias significativas entre la velocidad de los vehículos ligeros y los camiones ligeros, pero sí entre estos y los vehículos pesados (más de 2 ejes).

Asimismo, Saifizul et al. (2011) concluyeron que el tipo de vehículo tiene un efecto significativo en la velocidad pero solo en el caso en que el tamaño de los vehículos es significativamente diferente. En el caso en que los vehículos son similares en tamaño pero difieren en número de ejes, el peso es el factor dominante. Por otra parte, observaron que la velocidad se reduce de forma monótona al aumentar el peso bruto del vehículo para menores de $20 \mathrm{t}$, mientras que la velocidad se mantiene constante para mayores de este valor. 
Jacob y Anjaneyulu (2013) también observaron una diferencia significativa entre la velocidad de los vehículos ligeros y la de los pesados. Para un radio dado, la diferencia entre las velocidades de diferentes vehículos aumenta al aumentar la longitud de la curva. Asimismo, para una longitud de curva esta diferencia aumenta al aumentar el radio.

El estudio de las diferencias de velocidad de operación entre vehículos ligeros y pesados se ha extendido también a las variables que influyen en su elección. La mayor parte de las investigaciones con este objetivo concluyeron que, mientras que el perfil de velocidad de los vehículos ligeros puede basarse principalmente en el trazado en planta de la carretera, el perfil de velocidad de los vehículos pesados depende en gran medida también del trazado en alzado. En este sentido, Leisch y Leisch (1977) desarrollaron un perfil de velocidad combinando el perfil estimado considerando sólo el trazado vertical (asumiendo terreno llano) y el perfil estimado considerando sólo el trazado vertical (sin restricciones en el trazado horizontal).

La mayor parte de los modelos calibrados hasta el momento se han basado en una muestra relativamente reducida, por la dificultad de encontrar tramos de carreteras con una alta intensidad de pesados.

Donnell et al. (2001) basó su estudio en 17 curvas de carreteras convencionales. Completaron su estudio con datos procedentes de la simulación con el software TWOPAS. Tras el estudio concluyeron que el aumento del radio de la curva y la longitud de la recta anterior estaba asociado con una $\mathrm{V}_{85}$ P mayor en el punto de tangencia entre ambos elementos (PT), mientras que un aumento de la pendiente de la recta de entrada lo estaba con una disminución de la $\mathrm{V}_{85 \mathrm{P}}$ a lo largo de la recta hasta el punto PT. Por otra parte, un aumento de la pendiente de la recta de salida combinada con un aumento de la longitud de la misma recta se asoció con una disminución de la $\mathrm{V}_{85}$.

Gibreel et al. (2001) calibraron un modelo para la estimación de la velocidad de operación desarrollada en carreteras convencionales, sin distinguir entre vehículos ligeros y pesados. Utilizaron dos tipos de combinaciones de trazado: curva en planta con acuerdo cóncavo (9 tramos) y curva en planta con acuerdo convexo (10 tramos). Los resultados mostraron que las variables geométricas con mayor influencia en la velocidad de operación son: el radio de la curva, el ángulo de deflexión de la curva, la distancia horizontal entre el punto de intersección de las rectas y el vértice del acuerdo, longitud del acuerdo, pendientes, diferencia algebraica entre pendientes y el peralte.

Jacob y Anjaneyulu (2013) basaron su estudio en 147 curvas en carreteras con pendientes entre $-2 \%$ y $+2 \%$, por lo que no pudieron medir adecuadamente el efecto de la pendiente longitudinal en la velocidad de operación. Centrándose en las variables geométricas del trazado en planta, observaron que la velocidad de los vehículos pesados en recta aumentaba al aumentar la longitud de esta y que en curva la velocidad dependía tanto del radio como de 
la longitud de esta, aumentando con el radio y disminuyendo con la longitud.

Otros estudios no se han basado en la velocidad de operación de los vehículos pesados, sino

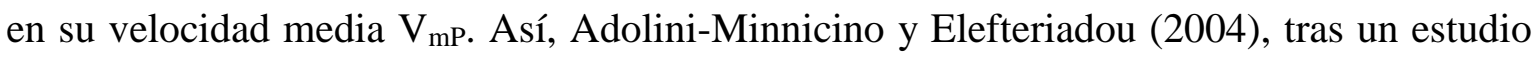
basado en datos de simulación con TWOPAS, concluyeron que un aumento de la longitud de la recta anterior y de su pendiente se asociaban a una disminución de esta velocidad en varios puntos entre el punto de curvatura (PC) y el punto de tangencia (PT) y que un aumento de la longitud del acuerdo vertical se asociaba a una disminución de $\mathrm{V}_{\mathrm{mP}}$.

Por otra parte, existen varios estudios que no se basan en datos experimentales, sino que se centran en el estudio del perfil de velocidad de operación de vehículos pesados en tramos de pendiente positiva a partir de un modelo dinámico-mecánico (Bester, 2000; Lan y Menendez, 2003; Echaveguren y Carrasco, 2015). Estos modelos tratan de modelizar la velocidad de estos vehículos a partir de la potencia del vehículo y la resistencia del aire, la pendiente, el rozamiento e incluso las condiciones del pavimento, asumiendo que los camiones disminuyen su velocidad hasta un cierto valor que mantienen constante hasta el final del tramo ascendente. Sin embargo, Arellano et al. (2015) identificaron que cuando la longitud del tramo ascendente era inferior a $1 \mathrm{~km}$, los camiones eran capaces de acelerar en el último tercio del ascenso.

Teniendo en cuenta el pequeño número de modelos calibrados en las investigaciones anteriores para la estimación de la velocidad de operación de vehículos pesados y la muestra en la que están basados los existentes, la presente investigación estudia la velocidad de operación de vehículos pesados en curvas de carreteras convencionales. Este estudio se basa en perfiles continuos de velocidad obtenidos mediante GPS ubicados en los vehículos de dos empresas distintas.

\section{OBJETIVOS E HIPÓTESIS}

El principal objetivo de esta investigación es analizar la distribución de la velocidad en flujo libre de vehículos pesados en curvas de carreteras convencionales, tomando como base datos experimentales. El estudio no se ha centrado únicamente en la velocidad de operación, entendida como el percentil 85 de la distribución de velocidad en flujo libre, sino en el estudio de la distribución completa. Se precisa este estudio completo ya que los percentiles bajos, como el percentil 15, están relacionados con el riesgo de alcance de vehículos ligeros.

La hipótesis en la que se basa este estudio es que tanto la media como la desviación típica de la distribución de velocidades de vehículos pesados es inferior a las de la distribución de velocidades de vehículos ligeros y, por tanto, los modelos calibrados para la estimación de la velocidad desarrollada por vehículos ligeros no son válidos para vehículos pesados.

Por otro lado, las variables con influencia en la elección de la velocidad también son 
distintas. La pendiente longitudinal de la carretera, que tiene una influencia mínima en los vehículos ligeros, produce que los vehículos pesados tengan altas tasas de deceleración y muy bajas velocidades en pendientes positivas prolongadas. Además, mientras que en los vehículos ligeros la elección de la velocidad depende más del conductor, en los vehículos pesados la velocidad depende principalmente de las características mecánicas de estos.

\section{METODOLOGÍA}

Esta investigación se basa en perfiles continuos de velocidad de vehículos pesados circulando en flujo libre, gracias a la colaboración de la empresa SAEVI, S.L. y a la empresa SAV - Agricultores de la Vega de Valencia. La empresa SAEVI, S.L. está dedicada a la extracción y venta de caolín, arcilla y productos derivados por lo que los vehículos que participaron en el estudio fueron vehículos pesados tipo bañera, mientras que los vehículos de la empresa SAV que participaron en el estudio fueron vehículos pesados utilizados para el transporte de residuos a vertedero, con remolque tanto tipo bañera como semirremolque.

Para la grabación de los datos se facilitó a las empresas colaboradoras una serie de dispositivos GPS de $1 \mathrm{~Hz}$ de pequeño tamaño que, gracias al potente imán que poseen, pueden colocarse en el exterior de la cabina de los vehículos, disminuyendo así la influencia sobre el comportamiento de los conductores. Las empresas colocaron estos GPS en los vehículos de su flota, registrando de forma continua la velocidad desarrollada por los vehículos durante la realización de su ruta habitual (Figura 1).

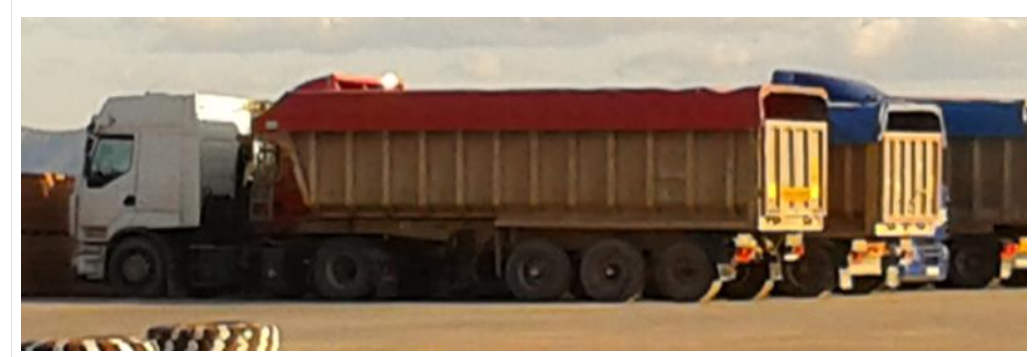

Fig. 1 - Tipo de vehículos utilizados en el estudio

La ruta de los vehículos seleccionados se extiende a varias carreteras de montaña con diferencias de trazado, tanto en planta como en alzado. De los tramos de los que se dispone datos se han elegido cuatro de ellos (Tabla 1) para el análisis que se presenta a continuación.

\begin{tabular}{|c|c|c|c|c|}
\hline Tramo & Carretera & Inicio & Final & Longitud (m) \\
\hline SAEVI_1 & CV-345 & Casinos & Villar del Arzobispo & $9.562,31$ \\
\hline SAEVI_2 & CV-345 & Villar del Arzobispo & Higueruelas & $7.461,22$ \\
\hline SAV_1 & CV-425 & Buñol & Alborache & $1.955,71$ \\
\hline SAV_2 & CV-425 & Macastre & Intersección CV-580 & $11.997,06$ \\
\hline
\end{tabular}

Tabla 1 - Tramos de estudio 
La geometría en planta de estos tramos se ha restituido mediante el algoritmo desarrollado por Camacho-Torregrosa et al. (2015) basado en el azimut. Por su parte, la geometría en alzado se ha restituido mediante el software para diseño geométrico de carreteras Civil 3D.

Sobre los trazados restituidos se han proyectado los perfiles continuos de velocidad de cada uno de los vehículos estudiados. Teniendo en cuenta que el control sobre el recorrido realizado por los mismos y sus condiciones de circulación fueron mínimas, es necesario estudiar cada uno de estos perfiles para garantizar que se incluyen en el estudio únicamente aquellos circulando en flujo libre. La metodología utilizada para la evaluación de las condiciones de circulación en flujo libre ha sido la desarrollada por Pérez-Zuriaga et al. (2013). Está basada en la hipótesis de que cada conductor se comporta de una forma particular, aproximándose su perfil de velocidad individual al de un percentil. Por tanto, cuando el perfil individual de un conductor se aleja bruscamente del percentil próximo se considera que está condicionado.

Una vez estudiada la geometría de los tramos de estudio y los perfiles continuos de velocidad desarrollados por los conductores en estos tramos, se consideró la opción de no considerar en el estudio los tramos Casinos - Villar del Arzobispo dirección Casinos, con tan solo 5 trayectorias, y el tramo Villar del Arzobispo - Higueruelas dirección Higueruelas, con tan solo 8 trayectorias. Sin embargo, sí que se han incluido en el estudio tras comprobar, como se verá en los siguientes apartados, que la dispersión de las velocidades de vehículos pesados en curvas es muy baja.

De esta forma, el número de curvas aisladas utilizadas en el estudio asciende a 69, evitando incluir en el estudio curvas compuestas. Dos de ellas presentan un radio de $796 \mathrm{~m}$, valor muy superior al siguiente radio $(428 \mathrm{~m})$, por lo que fueron excluidas del estudio, quedando un total de 67 curvas aisladas. Un resumen de sus características se muestra en la Tabla 2.

\begin{tabular}{|c|c|c|c|c|c|}
\hline & Radio $(\mathrm{m})$ & Longitud $(\mathrm{m})$ & CCR $(\mathrm{gon} / \mathrm{Km})$ & Pendiente $(\%)$ & \#Conductores \\
\hline Min. & 25 & 47,170 & 100,393 & $-0,0797$ & 5 \\
\hline Máx. & 428 & 182,716 & $2.077,357$ & 0,0797 & 135 \\
\hline
\end{tabular}

Tabla 2 - Resumen de las características de las curvas

\section{DISTRIBUCIÓN DE VELOCIDAD EN CURVAS}

El tratamiento de los datos de geometría y de velocidad, obtenidos de la toma de datos y de la restitución geométrica, dio como resultado una base de datos en la que para cada curva se dispone de sus características geométricas, la velocidad en flujo libre individual de cada uno de los conductores y la media, la desviación típica y los diferentes percentiles de la distribución de velocidad en flujo libre. 


\subsection{Media y desviación típica de la distribución de velocidad en curvas.}

Con el fin de estudiar la distribución de la velocidad desarrollada por vehículos pesados circulando por curvas en condiciones de flujo libre, se ha analizado el comportamiento de su media y su varianza.

La Figura 2 muestra cómo la media de la velocidad en flujo libre desarrollada por los vehículos pesados varía aumentando conforme aumenta el radio de la curva. Como se esperaba, las curvas con radio más pequeño presentan velocidades más bajas, mientras que curvas con radios mayores presentan mayores velocidades. La relación entre la velocidad media y el radio no es lineal, sino que para radios altos la velocidad se aproxima a una constante.

Asimismo, también se observa cómo los vehículos cargados tienen una velocidad ligeramente inferior a los vehículos descargados a igualdad de radio. Al presentarse dos poblaciones tan claramente definidas, el estudio las ha considerado por separado.

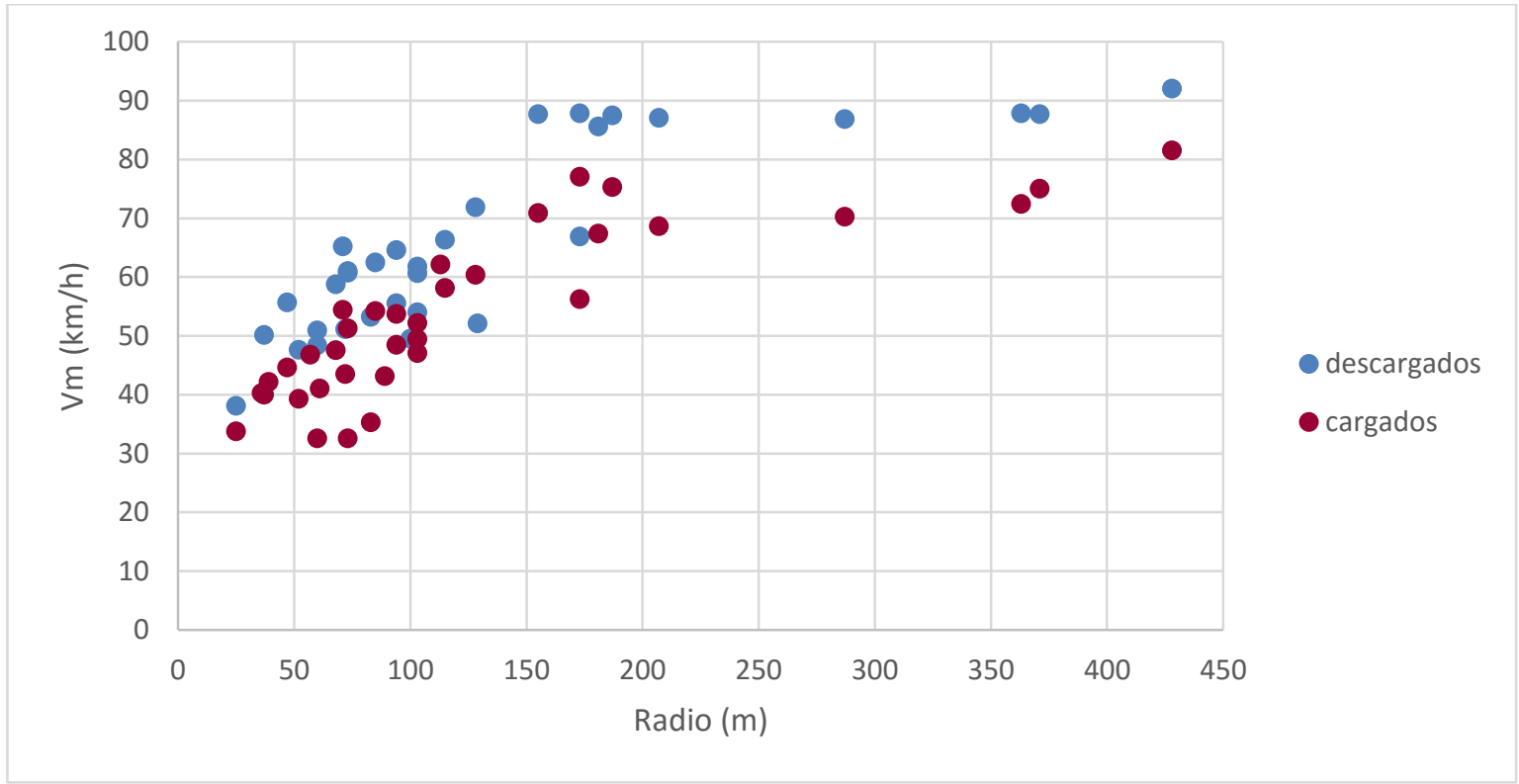

Fig. 2 - Velocidad media vs radio

Con el $90 \%$ de estos datos se han calibrado sendos modelos que permiten la estimación de la velocidad media de vehículos pesados en curvas en función del radio de las mismas. El $10 \%$ restante se ha reservado para la validación de los mismos.

La calibración del modelo ha dado como resultado las Ecuaciones 1 y 2.

$$
\begin{array}{ll}
V_{m P d}=97.228-68.619 / e^{0.00691 \cdot R} & \mathrm{R}^{2}=0.078 \\
V_{m P c}=81.913-59.192 / e^{0.00723 \cdot R} & \mathrm{R}^{2}=0.079
\end{array}
$$

Donde:

$V_{m P d}:$ velocidad media de pesados descargados $(\mathrm{km} / \mathrm{h})$ 
$V_{m P c}:$ velocidad media de pesados cargados $(\mathrm{km} / \mathrm{h})$

$R$ : radio de la curva (m)

La Figura 3 muestra tanto la representación de las ecuaciones del modelo calibrado como los resultados de la validación. En ella, puede observarse como el modelo se ajusta adecuadamente a la velocidad desarrollada por los vehículos pesados, tanto cargados como descargados, en curvas de carreteras convencionales.

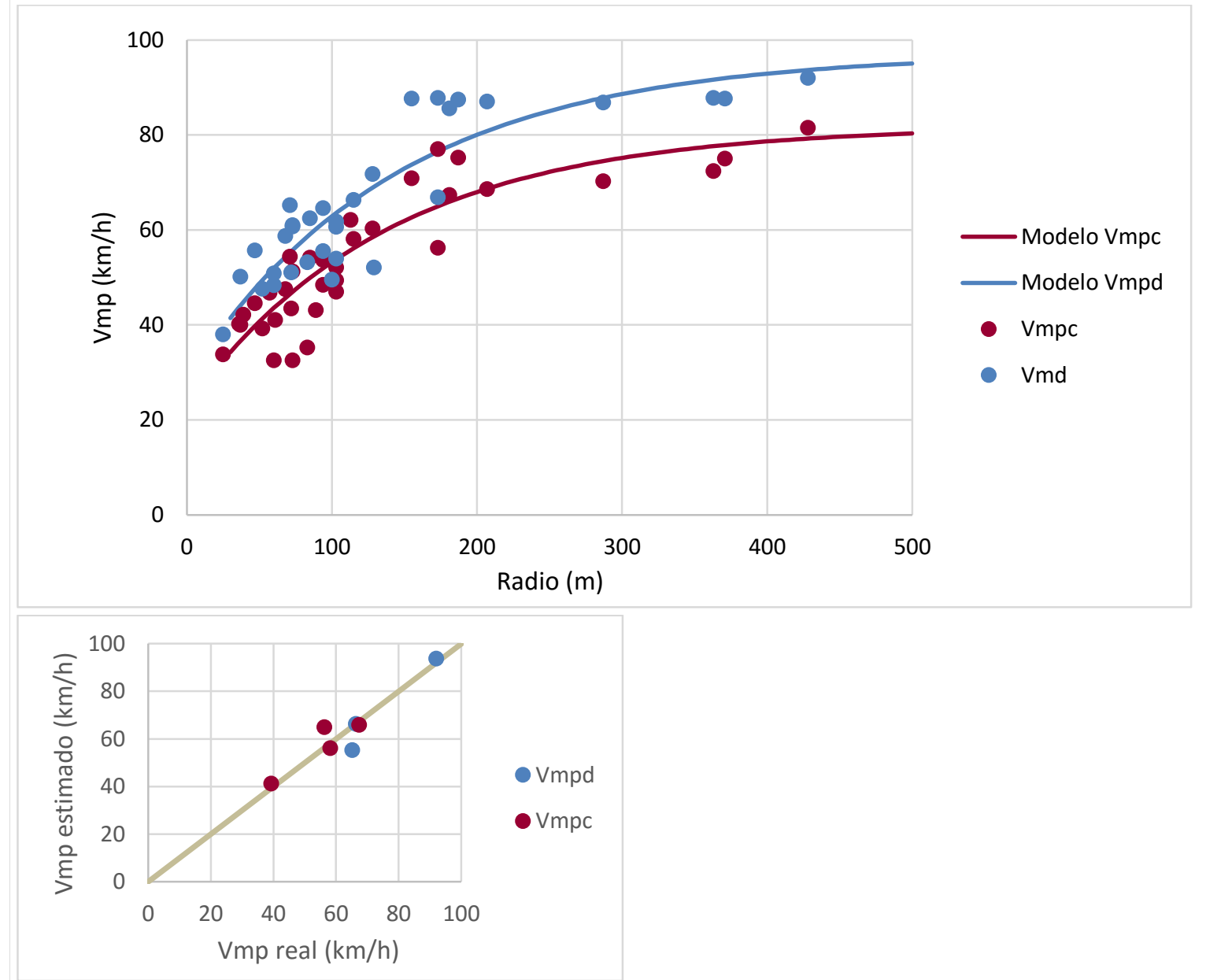

Fig. 3 - a. Modelo para la estimación de $V_{\text {mp }}$ b. Validación del modelo $V_{\text {mp }}$

Adicionalmente, se ha realizado un estudio de la desviación típica de la distribución. Como se observa en la Figura 4, el comportamiento de la desviación típica no presenta ninguna tendencia apreciable respecto al radio de la curva. Este análisis se ha realizado con otras variables, incluida la pendiente, y el resultado ha sido similar: los valores de la desviación típica de la distribución de la velocidad en cada una de las curvas varían entre $0,5 \mathrm{~km} / \mathrm{h}$ y $3,5 \mathrm{~km} / \mathrm{h}$ (salvo dos valores extremos), tanto para vehículos cargados como para vehículos descargados. 


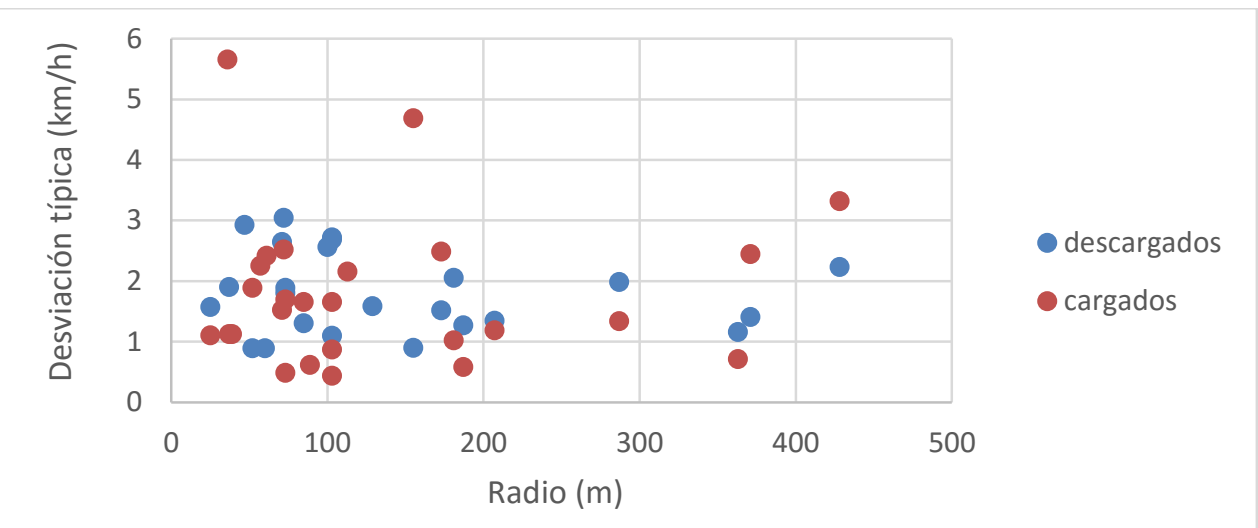

Fig. 4 - Desviación típica vs radio

Considerando estos valores de desviación típica, con un tamaño muestral de 5 vehículos (la menor del estudio), se obtendría un error muy pequeño con un nivel de confianza del $95 \%$, por lo que la decisión de incluirlos en el estudio puede considerarse válida.

\subsection{Percentil 85 .}

Como se ha puesto de relieve en el apartado de introducción con la revisión de las investigaciones anteriores, el parámetro utilizado para la evaluación de la consistencia del diseño geométrico es el percentil 85 de la distribución de velocidades en flujo libre. Por ello, la mayor parte de los modelos y análisis previos se han centrado en este percentil.

A partir de los datos tomados en campo y posteriormente tratados, se ha seleccionado un 90\% de los datos de forma aleatoria para la calibración de un modelo que estime la velocidad de operación de los vehículos pesados $\left(\mathrm{V}_{85}\right)$ en función de las características del trazado, tanto en planta como en alzado. El 10\% restante se han dedicado a la validación del mismo.

Al igual que Leisch y Leisch (1977), en un primer momento se planteó la posibilidad de desarrollar un modelo combinado compuesto por un modelo que considere únicamente el trazado vertical y un modelo que considere únicamente el trazado horizontal. La velocidad estimada resultado del modelo combinado sería la menor de las velocidades resultantes de los modelos constituyentes. Sin embargo, tras el estudio de la influencia de la pendiente en la elección de la velocidad en curvas (Figura 5) se optó por no tenerla en cuenta y calibrar el modelo considerando únicamente parámetros relacionados con el trazado en planta. 


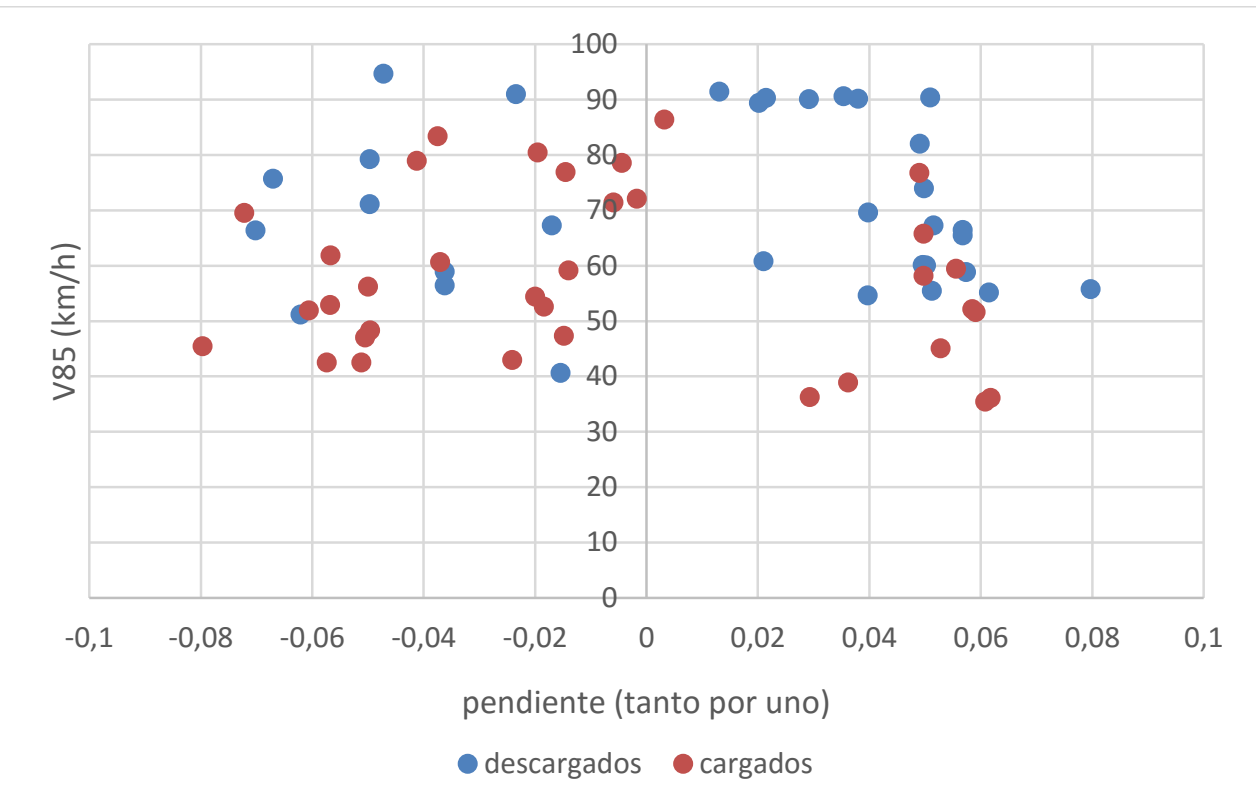

Fig. 5 - V85P vs pendiente

Tras el estudio de la influencia de varias variables relacionadas con el trazado en planta y la calibración de diferentes ecuaciones, se ha concluido que el modelo que mejor estima el comportamiento de los vehículos pesados en curvas de carreteras convencionales es que el que viene definido por las Ecuaciones 3 y 4.

$$
\begin{array}{ll}
V_{85 P d}=98.627-64.061 / e^{0.00744 \cdot R} & \mathrm{R}^{2}=0.075 \\
V_{85 P c}=86.453-62.417 / e^{0.00779 \cdot R} & \mathrm{R}^{2}=0.079
\end{array}
$$

Donde:

$V_{85 P d}$ : percentil 85 de la distribución de velocidades de pesados descargados $(\mathrm{km} / \mathrm{h})$ $V_{85 P c}$ : percentil 85 de la distribución de velocidades de pesados cargados $(\mathrm{km} / \mathrm{h})$ $R$ : radio de la curva $(\mathrm{m})$

La Figura 6 incluye tanto la representación del modelo como los resultados de la validación del mismo, mostrando como el modelo se ajusta adecuadamente al percentil 85 de la distribución de velocidad desarrollada por vehículos pesados, tanto cargados como descargados, circulando en condiciones de flujo libre por curvas de carreteras convencionales. 

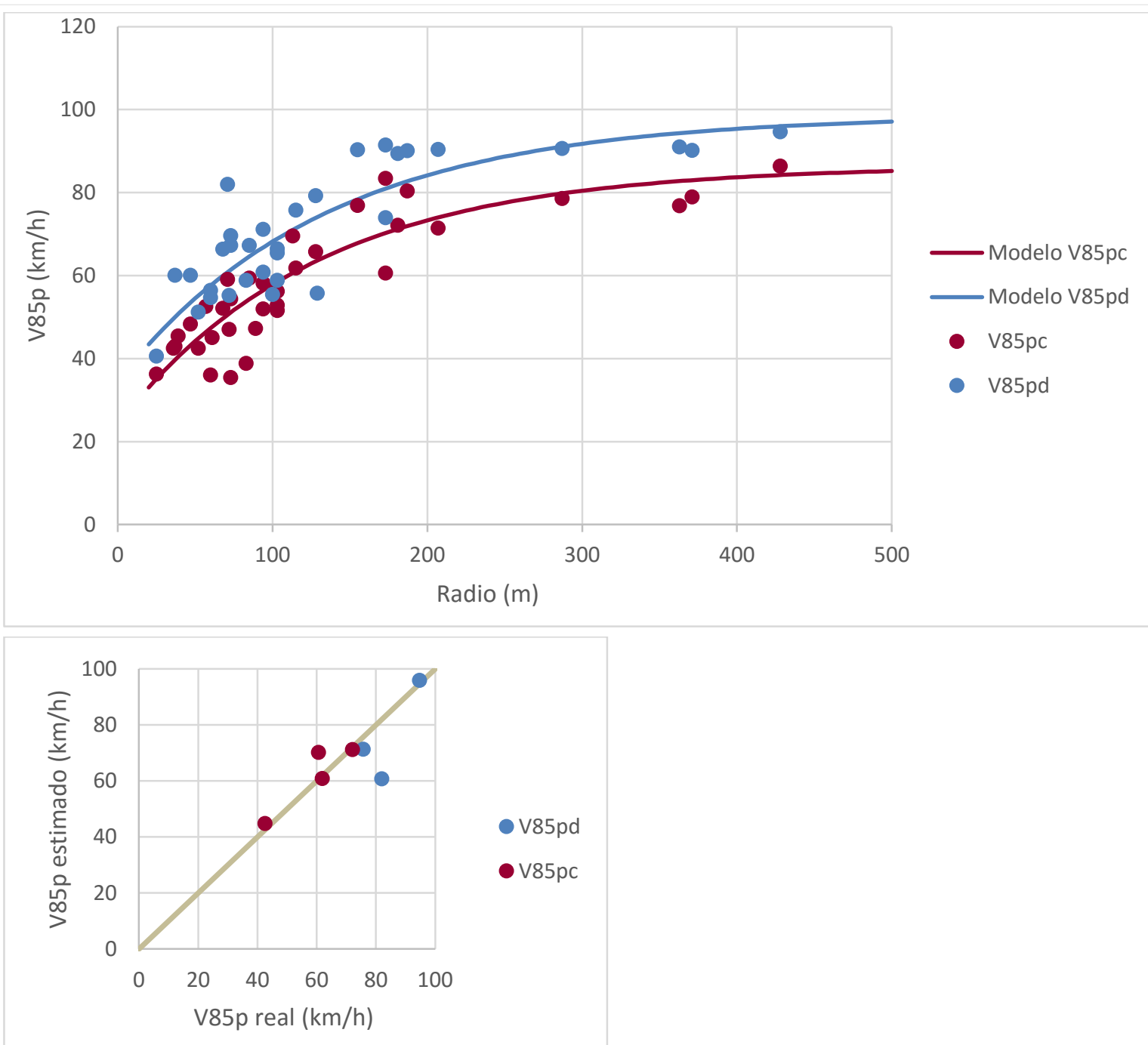

Fig. 6 - a. Modelo para la estimación de V85p b. Validación del modelo V85p

\subsection{Percentil 15.}

Como se ha descrito en apartados anteriores, es fundamental tener los medios para poder estimar el percentil 85 de la distribución de velocidad de los vehículos pesados para poder obtener una mejor evaluación de la consistencia de un trazado. Sin embargo, en cuanto a la funcionalidad del tráfico los percentiles que más influyen son los percentiles más bajos, pues son estos los que están relacionados con el riesgo de alcance de vehículos ligeros. Por ello, en este estudio no sólo se ha calibrado un modelo para la estimación del percentil 85 ( $\mathrm{V}_{85 \mathrm{P}}$ ), sino también un modelo para la estimación del percentil $15\left(\mathrm{~V}_{15 \mathrm{P}}\right)$.

Como en el caso anterior, se ha evaluado tanto el trazado en planta como el trazado en alzado $y$, al igual que en el caso anterior, se ha comprobado que el control de la velocidad viene dado por el trazado en planta, no teniendo especial influencia la pendiente longitudinal en la elección de la velocidad. Por tanto, los modelos estudiados se han centrado en parámetros relacionados con el trazado en planta. Tras este estudio, se ha concluido que las ecuaciones que mejor ajustan el $\mathrm{V}_{15 \mathrm{P}}$ son las Ecuaciones 5 y 6. 


$$
\begin{array}{ll}
V_{15 P d}=91.546-67.827 / e^{0.007006 \cdot R} & \mathrm{R}^{2}=0.078 \\
V_{15 P c}=78.855-56.742 / e^{0.00618 \cdot R} & \mathrm{R}^{2}=0.079
\end{array}
$$

Donde:

$V_{15 P d}:$ percentil 15 de la distribución de velocidades de pesados descargados $(\mathrm{km} / \mathrm{h})$

$V_{15 P c}$ : percentil 15 de la distribución de velocidades de pesados cargados $(\mathrm{km} / \mathrm{h})$

$R$ : radio de la curva (m)

La Figura 7 muestra la representación del modelo y los resultados de su validación, mostrando como el modelo se ajusta adecuadamente al percentil 15 de la distribución de velocidad desarrollada por vehículos pesados, tanto cargados como descargados.

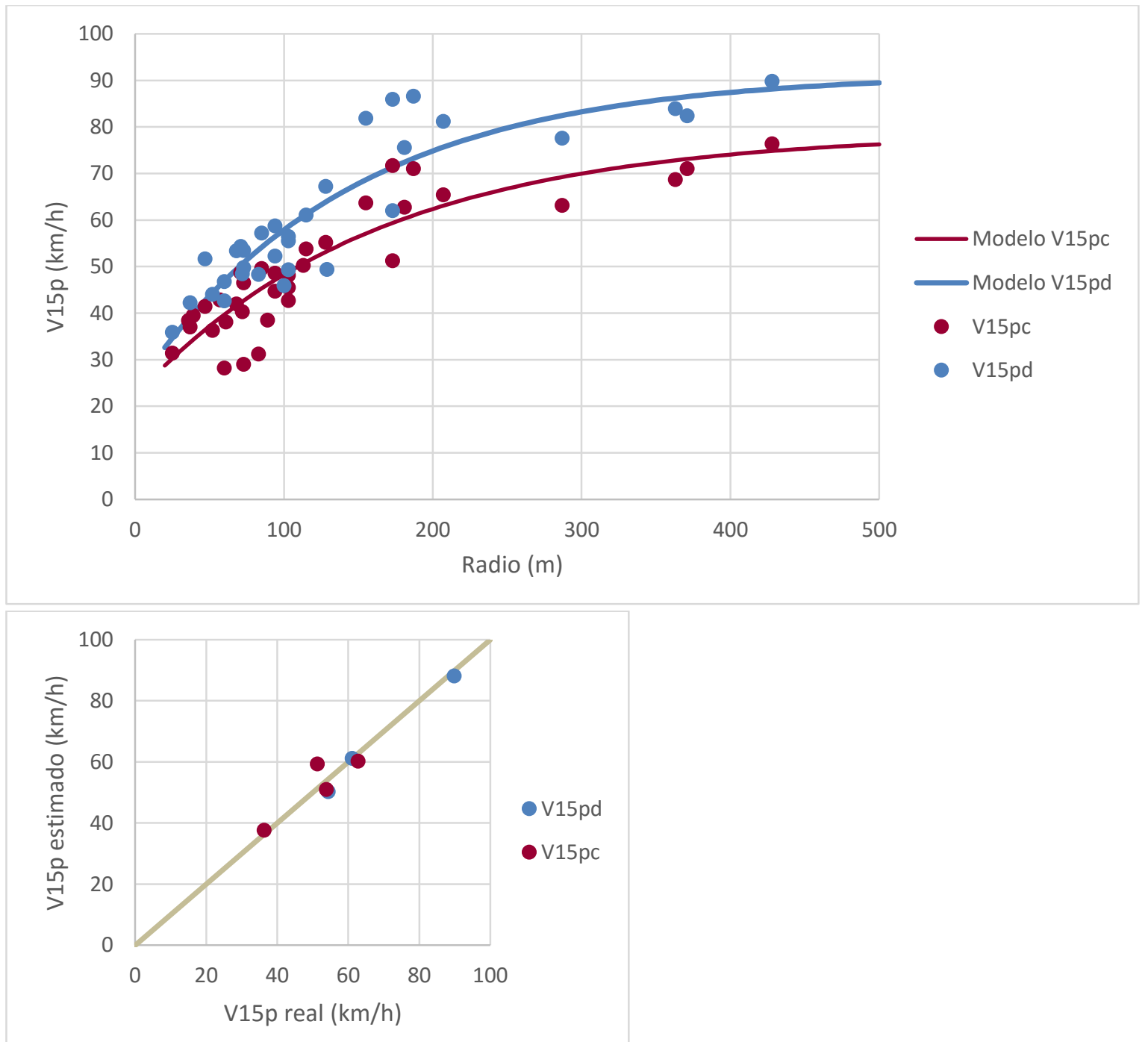

Fig. 7 - a. Modelo para la estimación de $V_{15 p}$ b. Validación del modelo V15p

\section{DISCUSIÓN}

El análisis de los estudios existentes relacionados con la velocidad desarrollada por los vehículos pesados circulando en condiciones de flujo libre por carreteras convencionales 
plantea una serie de hipótesis que van a ser analizadas en el presente apartado.

\subsection{Influencia de la pendiente en la velocidad de los vehículos pesados.}

Como se ha mostrado en el apartado de introducción, la mayor parte de las investigaciones anteriores han tomado como hipótesis o han concluido que las variables con influencia en la elección de la velocidad entre vehículos ligeros y pesados son distintas. Especialmente en lo que se refiere a la influencia de la pendiente. Se entiende que, mientras que su influencia es mínima en los vehículos ligeros, el aumento de la pendiente produce una disminución clara de la velocidad de los vehículos pesados.

En el estudio que se presenta, se ha comprobado que, en curvas de carreteras convencionales, esta hipótesis no es cierta. Como se observa en la Figura 5, la influencia de la pendiente en la velocidad desarrollada por los vehículos pesados, tanto cargados como descargados, es mínima en curvas de carreteras convencionales. Es posible que ello se deba a que, aunque la pendiente sí influye en la elección de la velocidad, el principal control en curvas es el radio de las mismas.

Es futuras investigaciones se analizará la influencia de la pendiente en la elección de la velocidad de vehículos pesados circulando a lo largo de una recta, donde el control geométrico del trazado en planta es mínimo.

\subsection{Velocidad de vehículos ligeros vs velocidad de vehículos pesados.}

Una de las principales hipótesis en las que se basan los estudios relacionados con vehículos pesados es que tanto la media como la desviación típica de la distribución de velocidades de vehículos pesados es inferior a las de la distribución de velocidades de vehículos ligeros y, por tanto, los modelos calibrados para la estimación de la velocidad desarrollada por vehículos ligeros no son válidos para vehículos pesados.

En el estudio que se presenta se ha comprobado que, efectivamente, los modelos calibrados para la estimación de la velocidad desarrollada por vehículos ligeros no son válidos para vehículos pesados. Sin embargo, la hipótesis que fundamenta este hecho no es cierta, según los datos experimentales estudiados.

El modelo desarrollado para la estimación de la velocidad media de vehículos pesados $\left(\mathrm{V}_{\mathrm{mP}}\right)$ se ha comparado con el modelo desarrollado por García-Jiménez et al. (2016) para vehículos ligeros $\left(\mathrm{V}_{\mathrm{mL}}\right)$ en la Figura 8. En ella se observa cómo los vehículos ligeros desarrollan una velocidad intermedia entre la desarrollada por los vehículos pesados cargados y la desarrollada por los descargados. La explicación a este fenómeno viene dada por el hecho de que los conductores de vehículos pesados son conductores profesionales con rutas recurrentes, por lo que tienen tanto un alto conocimiento del vehículo como de la carretera por la que circulan. Ello conlleva que cuando el vehículo va descargado las velocidades desarrolladas sean mayores que las de vehículos ligeros, donde las características de los 
conductores son más variadas.

Sin embargo, cuando el vehículo está cargado, el factor limitante es el peso del vehículo, lo que hace que las velocidades se reduzcan considerablemente, siendo inferiores a las de los vehículos ligeros.

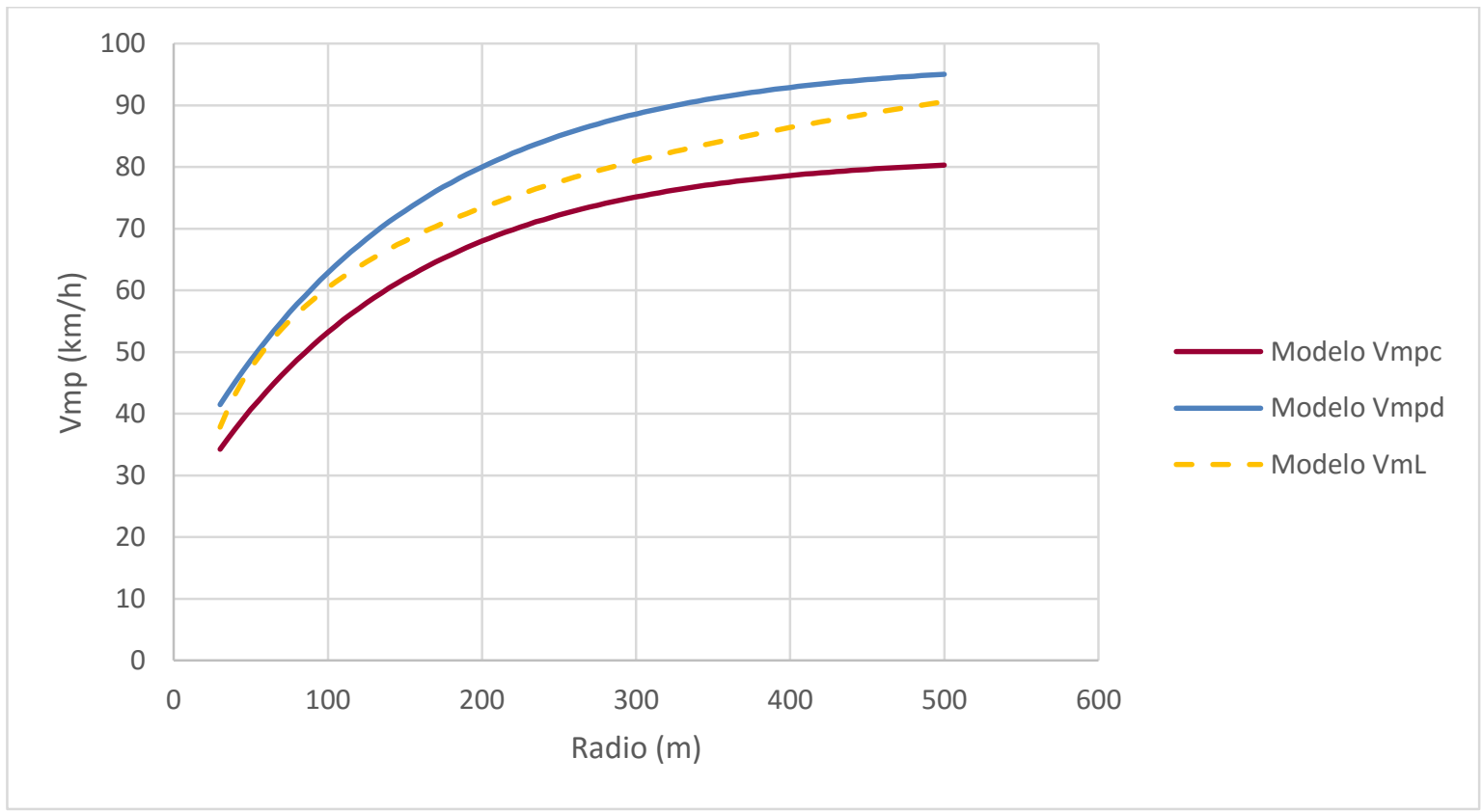

Fig. 8 - V $\mathbf{m P}$ vs $V_{m L}$

Adicionalmente, se ha estudiado también la desviación típica (Figura 4), concluyendo que su valor es mínimo y no depende del radio de la curva. Esto es debido a las características de los conductores de estos vehículos. Mientras que los conductores de los vehículos ligeros tienen características muy diversas, lo que hace que la desviación típica de la distribución de velocidades sea mayor, los conductores de vehículos pesados son profesionales con características similares, con lo que la dispersión de velocidades es mucho menor.

En el caso de los vehículos ligeros, la dispersión aumenta con el radio, ya que para radios mayores el control geométrico es menor, cobrando importancia las características de los conductores. En el caso de los vehículos pesados, no se ha observado esta tendencia, manteniéndose la desviación típica prácticamente constante.

\section{CONCLUSIÓN}

El conocimiento de la velocidad desarrollada por los vehículos pesados en carreteras convencionales es esencial tanto para la evaluación de la consistencia del diseño geométrico como para el estudio de la funcionalidad del tráfico. Sin embargo, rara vez se considera su efecto debido a la inexistencia de modelos que permitan la estimación del percentil 85 de su distribución de velocidad para la evaluación de la consistencia y del percentil 15 para la 
evaluación de su funcionalidad.

En este artículo se han calibrado y validado sendos modelos para la estimación de los percentiles citados en función de las características geométricas de la carretera, considerando tanto el trazado en planta como en alzado.

Su desarrollo se ha basado en datos de velocidad en el centro de 67 curvas situadas en cuatro tramos de carreteras convencionales desarrollada por vehículos pesados circulando en flujo libre. Para la toma de datos se ha contado con la colaboración de las empresas SAEVI y $\mathrm{SAV}$, que, desinteresadamente, permitieron la colocación de un dispositivo GPS de $1 \mathrm{~Hz}$ en su flota de camiones. De esta forma, se grabaron los datos de velocidad de vehículos circulando en su ruta habitual.

Con estos datos se ha calibrado un modelo para la estimación del percentil 85 de la velocidad de vehículos pesados y otro para el percentil 15. Ambos están formados por dos ecuaciones, una para vehículos cargados y otra para vehículos descargados, dependiendo ambas únicamente del radio en planta. En ninguno de ellos se ha incluido ninguna variable relacionada con el trazado en alzado, ya que, tras un estudio de la influencia de la pendiente en la velocidad, se ha concluido que su influencia es mínima.

Adicionalmente, se ha estudiado también la evolución de la velocidad media y de la desviación típica en función de las características geométricas del trazado en planta, con el fin de compararla con la de vehículos ligeros. De este análisis se ha concluido que, en cuanto a velocidad, los vehículos ligeros desarrollan una velocidad intermedia entre la desarrollada por los vehículos pesados cargados y la desarrollada por los vehículos pesados descargados. En cuanto a la desviación típica, en el caso de los vehículos ligeros la dispersión de las velocidades depende del radio, aumentando con él, mientras que, en el caso de vehículos pesados, la dispersión se mantiene prácticamente constante en un valor muy reducido.

Por tanto, se puede concluir que los vehículos pesados no se comportan igual que los vehículos ligeros, por lo que es esencial considerarlos por separado.

Por ello, la calibración y validación de los modelos presentados para la estimación de $\mathrm{V}_{85} \mathrm{P}$ y $V_{15 P}$ pueden suponer un punto de partida para la consideración de este tipo de vehículos tanto en la evaluación de la consistencia del diseño geométrico como en la de la funcionalidad del tráfico. De esta forma se mejoraría considerablemente tanto la seguridad vial como la operación vehicular de las carreteras convencionales.

\section{AGRADECIMIENTOS}

El estudio que se ha presentado es parte del proyecto de investigación "CASEFU - Estudio experimental de la funcionalidad y seguridad de las carreteras convencionales" (TRA201342578-P), subvencionado por el Ministerio de Economía y Competitividad y el Fondo Social 
Europeo. Asimismo, los autores quieren agradecer a las empresas SAV y SAEVI, así como la Entidad Metropolitana para el Tratamiento de Residuos en Valencia, por su colaboración totalmente desinteresada en toma de datos.

\section{REFERENCIAS}

AASHTO (2011). A Policy on Geometric Design of Highways and Streets. American Association of State Highway and Transportation, EEUU.

ADOLINI-MINNICINO, M. y ELEFTERIADOU, L. (2004). Speed prediction models for trucks on two-lane rural highways. 83rd Annual Meeting of the Transportation Research Board, Washington, DC.

ARELLANO, D., ECHAVEGUREN, T. y VARGAS-TEJEDA, S. (2015). A model of truck speed profiles on short upward slopes. Transport, vol. 168, pp. 475-483.

BESTER, C. (2000). Truck speed profiles. Transportation Research Record: Journal of the Transportation Research Board, vol. 1701, pp. 111-115.

CAMACHO-TORREGrosA, F. J., PÉREZ-ZURIAGA, A. M., CAMPOY-UNGRÍA, J. M., GARCÍA, A. y TARKO, A. P. (2015). Use of Heading Direction for Recreating the Horizontal Alignment of an Existing Road. Computer-Aided Civil and Infrastructure Engineering, vol. 30(4), pp. 282-299.

DONNELL, E. T., NI, Y., ADOLINI, M., y ELEFTERIADOU, L. (2001). Speed prediction models for trucks on two-lane rural highways. Transportation Research Record: Journal of the Transportation Research Board, vol. 1751, pp. 44-55.

ECHAVEGUREN, T., y CARRASCO, D. (2015). Estimación de longitud crítica en pendientes ascendentes en caminos bidireccionales. Revista Ingeniería de Construcción, vol. 30(1), pp. 5-16.

GARCÍA-JIMÉNEZ, E., PÉREZ-ZURIAGA, A.M., CAMACHO-TORREGROSA, F.J., LLOPIS-CASTELLÓ, D. y GARCÍA, A. (2016). Velocidad de operación de vehículos ligeros en curvas de carreteras convencionales. XII Congreso de Ingeniería del Transporte, Valencia.

GIBREEL, G.M.; EASA, S.M. y EL-DIMEERY, I.A. (2001). Prediction of operating speed on three-dimensional highway alignment. Journal of Transportation Engineering, vol. 127 (1), pp. 21-30.

HARWOOD, D. W., TORBIC, D. J., RICHARD, K. R., GLAUZ, W. D., y ELEFTERIADOU, L. (2003). Review of truck characteristics as factors in roadway design. NCHRP Rep. 505, Transportation Research Board, Washington, DC.

JACOB, A. y ANJANEYULU M. V. L. R. (2013). Operating Speed of Different Classes of Vehicles at Horizontal Curves on Two-Lane Rural Highways. Journal of Transportation Engineering, vol. 139, pp. 287-294.

LAN, C. J., \& MENENDEZ, M. (2003). Truck speed profile models for critical length of 
grade. Journal of transportation engineering, vol. 129(4), pp. 408-419.

LEISCH, J. E., y LEISCH, J. P. (1977). New concepts in design speed application. Transportation Research Record: Journal of the Transportation Research Board, vol. 631, pp. 15-23.

MISAGHI, P. y HASSAN, Y. (2005). Modeling operating speed and speed differential on two-lane rural roads. Journal of transportation engineering, vol. 131, pp. 408-418.

PÉREZ-ZURIAGA, A. M., CAMACHO-TORREGROSA, F. J., GARCÍA, A. y CAMPOYUNGRÍA, J. M. (2013). Application of global positioning system and questionnaires data for the study of driver behaviour on two-lane rural roads. IET Intelligent Transport Systems, vol. 7 (2), pp. 182-189.

SAIFIZUL, A.A., YAMANAKA, H. y KARIM, M.R. (2011). Empirical analysis of gross vehicle weight and free flow speed and consideration on its relation with differential speed limit. Accident Analysis and Prevention, vol. 43, pp. 1068-1073. 